\title{
Far Lateral Transcondylar Transtubercular Approach for Microsurgical Resection of Foramen Magnum Meningioma: Operative Video and Technical Nuances
}

\author{
James K. Liu ${ }^{1} \quad$ Vincent N. Dodson ${ }^{1} \quad$ Ali T. Meybodi ${ }^{1}$
}

\author{
${ }^{1}$ Department of Neurological Surgery, Center for Skull Base and \\ Pituitary Surgery, Neurological Institute of New Jersey, Rutgers \\ University, New Jersey Medical School, Saint Barnabas Medical \\ Center, RWJ Barnabas Health, Newark and Livingston, New Jersey, \\ United States
}

\author{
Address for correspondence James K. Liu, MD, Department of \\ Neurological Surgery, Rutgers University, New Jersey Medical School, \\ Saint Barnabas Medical Center, RWJ Barnabas Health, 90 Bergen \\ Street, Suite 8100, Newark, NJ 07103, United States \\ (e-mail: james.liu.md@rutgers.edu).
}

J Neurol Surg B 2021;82(suppl S1):S19-S21.

\begin{abstract}
Surgical access to the ventral foramen magnum remains a technical challenge. With large lesions in this region compressing the brainstem and distorting the regional neurovascular relationships, formulating a surgical plan and its appropriate execution have crucial importance in achieving favorable outcomes. While the endoscopic endonasal approaches have gained increasing attention to access the clivus and the ventral brainstem, foramen magnum meningiomas are still preferred to be removed via an approach that obviates a trajectory through the nasopharyngeal mucosa. Therefore, the far lateral approach remains one of the most practical approaches for these challenging lesions. This operative video demonstrates the use of the far lateral transcondylar transtubercular approach to remove a

Keywords

- far lateral approach

- transcondylar transtubercular

- foramen magnum meningioma

- skull base surgery large meningioma in the ventral foramen magnum in a 63-year-old male with progressive cervical myelopathy, presenting as spastic quadriparesis without any cranial nerve abnormality. Using a right-sided far lateral transcondylar transtubercular approach, the meningioma was exposed within the cerebellomedullary gutter engulfing the vertebral artery and distorting the course of the adjacent cranial nerves. Using the different corridors identified between the vertebral artery, spinal accessory, vagus, and hypoglossal nerves, multiple angles of attacks to the tumor were established and utilized to resect the lesion. A
\end{abstract}

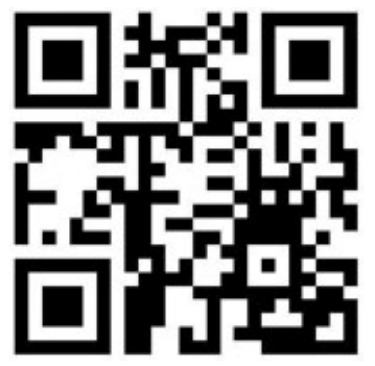

received

June 25,2020

accepted

June 25, 2020

published online

December 2, 2020

Conflict of Interest

None declared.

www.thieme.com/skullbasevideos

www.thieme.com/jnlsbvideos

DOI https://doi.org/

10.1055/s-0040-1716533. ISSN 2193-6331. (c) 2020. The Author(s).

This is an open access article published by Thieme under the terms of the Creative Commons Attribution-NonDerivative-NonCommercial-License, permitting copying and reproduction so long as the original work is given appropriate credit. Contents may not be used for commercial purposes, or adapted, remixed, transformed or built upon. (https://creativecommons.org/ licenses/by-nc-nd/4.0/) Georg Thieme Verlag KG, Rüdigerstraße 14, 70469 Stuttgart, Germany 

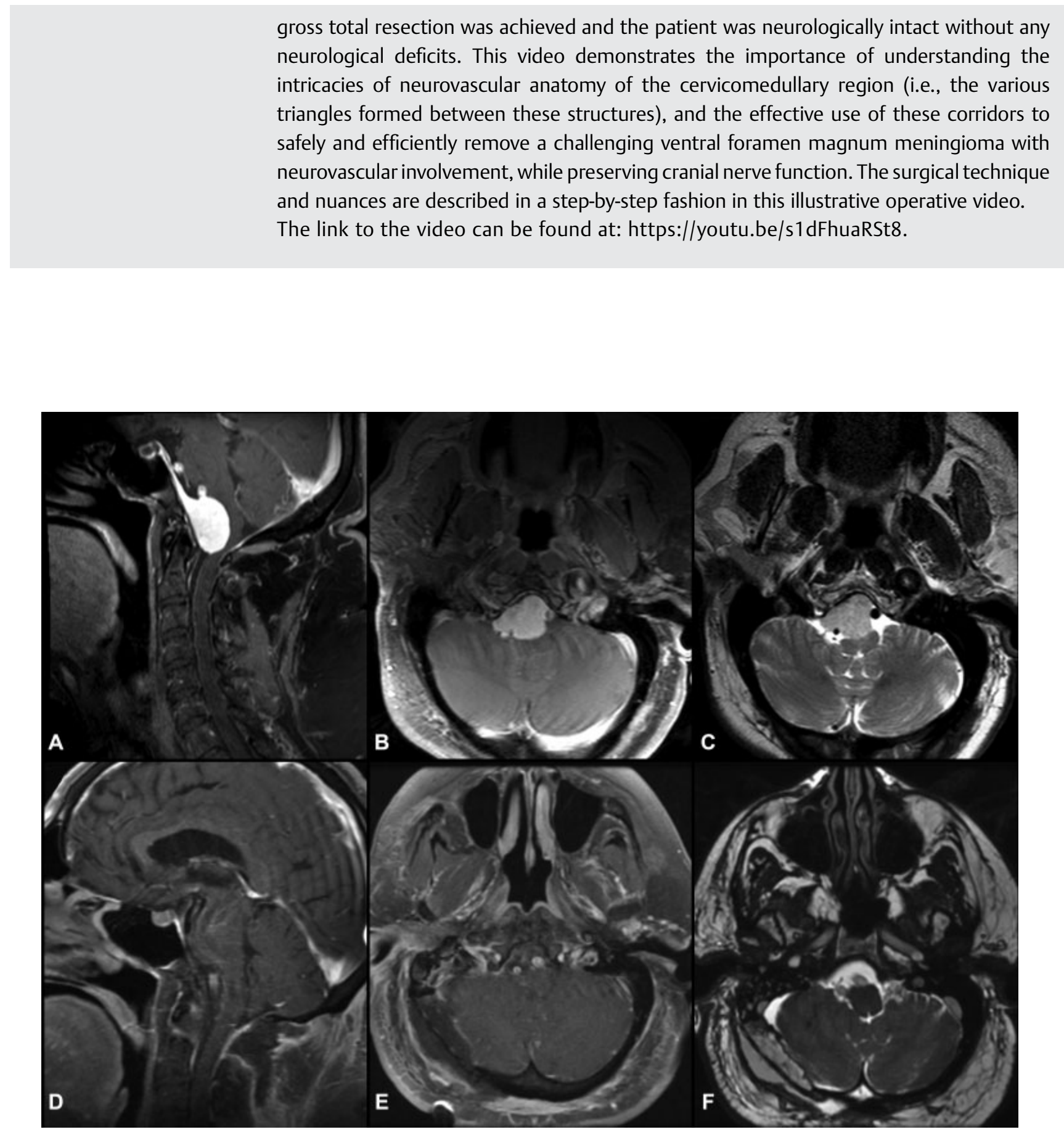

Fig. 1 (A-C) Preoperative MR imaging. (A) Sagittal gadolinium-enhanced T1-weighted, (B) axial gadolinium-enhanced T1-weighted, and (C) axial T2-weighted showing an extra-axial lesion located in the region of the foramen magnum and ventral to the lower brainstem, in the lower one-third of the clivus. The lesion is dural-based and shows bright enhancement and a prominent dural tail (A) consistent with a foramen magnum meningioma. Flow void signals on the either side of the lesion in the axial imaging (C) show close proximity of the lesion with bilateral vertebral arteries. (D-F) Postoperative MR imaging. (D) sagittal fat-suppressed postgadolinium T1-weighted, (E) axial fat-suppressed postgadolinium T1-weighted, and (F) axial T2-weighted show total resection of the lesion and satisfactory decompression of the brainstem with intact bilateral vertebral arteries ventral to the medulla. MR, magnetic resonance. 

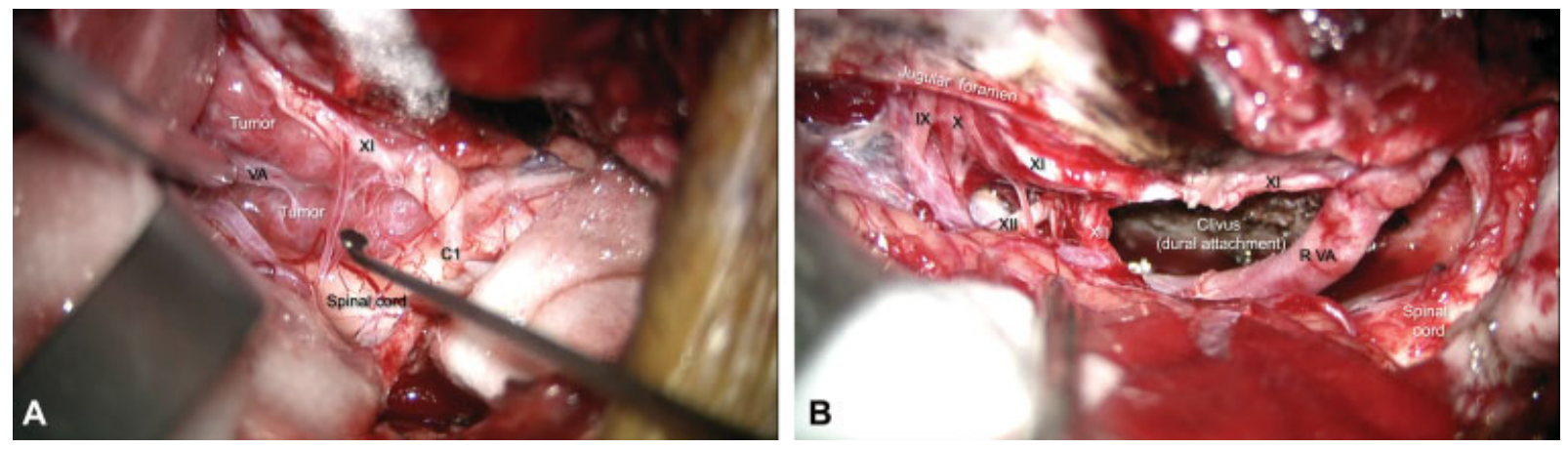

Fig. 2 (A) Intraoperative image showing the initial tumor exposure through a right far lateral transcondylar transtubercular approach with the distorted neurovascular anatomy. The tumor engulfs the right vertebral artery (VA) and compresses the cervicomedullary junction medially. The spinal accessory nerve (XI) is pushed posterolaterally. (B) Intraoperative image showing the surgical field after total resection of the tumor. The caudal cranial nerves are visualized. Note the 2 bundles of the hypoglossal nerve rootlets converging laterally to enter the hypoglossal canal. The ventral attachment of the tumor to the clival dura is coagulated to minimize the risk of recurrence. C1, C1 nerve root; R, right; IX, glossopharyngeal nerve; $\mathrm{X}$, vagus nerve; XII, hypoglossal nerve. 